\title{
Risk Factors Associated with Prolonged Hospital Stay in Admitted Patients with Psychotropic Drug Overdose
}

\author{
Toshikazu Abe ${ }^{*}, 1$ Yasuharu Tokuda ${ }^{2}$, Gerald H. Stein ${ }^{3}$, Shinichi Ishimatsu ${ }^{1}$ and Richard B. Birrer ${ }^{4}$ \\ ${ }^{I}$ Department of Emergency and Critical Care Medicine, St. Luke's International Hospital, Tokyo, Japan \\ ${ }^{2}$ Clinical Practice Evaluation and Research Center, St. Luke's Life Science Institute, Tokyo, Japan \\ ${ }^{3}$ Department of Medicine, College of Medicine, University of Florida, Gainesville, Florida, and University of Hawaii, \\ Honolulu, Hawaii, USA \\ ${ }^{4}$ Department of Emergency Medicine, Cornell University School of Medicine, New York, NY, USA
}

\begin{abstract}
Objective: To determine risk factors associated with their length of hospital stay in admitted patients with psychotropic drug overdose.

Methods: Demographics, data of physical and laboratory, and hospital stay were analyzed using a linear regression and a recursive partitioning analysis to determine significant risk factors for prolonged hospitalization.

Results: In 140 patients (mean age, $35 \mathrm{yr} ; 78 \%$ women) with psychotropic drug overdose, the median hospital stay was 2.9 days (range, 1-17) and all were discharged alive. Significant factors for prolonged hospital stay were older age $(p<0.0001)$, increased heart rate $(p=0.029)$, lower blood pressure $(p<0.0001)$, lower Glasgow coma scale $(p=0.015)$, and increased blood leukocyte $(\mathrm{p}=0.005)$. The recursive partitioning showed that blood leukocyte of $12,900 / \mathrm{mm}^{3} \mathrm{was}$ the cutoff of the most important variable for hospital stay of 3 days or greater.
\end{abstract}

Conclusions: Elevated blood leukocyte is the most important predictor for prolonged hospitalization in admitted patients with psychotropic drug overdose.

Keywords: Drug overdose, benzodiazepines, length of stay, risk factors, Japan.

\section{INTRODUCTION}

Problems related to intentional drug overdose (DO) are as common as in Tokyo, Japan, as in urban cities of other developed countries [1-3]. Patients with DO in other countries are involved with analgesics such as acetaminophen, opioids such as heroin and psychostimulants such as cocaine $[4,5]$, while patients with intentional drug overdose in Japan are usually involved with psychotropic drugs such as benzodiazepines, although there were no reports of epidemiology about patients with DO. Most episodes of DO in Japan are from psychotropic drugs and lead to presentation to emergency departments (ED) and admission to acute care hospitals. According to the database of Tokyo Fire Department managed by Tokyo prefecture government, 7152 patients with DO were identified in 2005 over an entire metropolitan area (unpublished data). These patients form a significant part of the workloads of urban EDs. Reports from the UK have also indicated that the similar degree of heavy workloads is expended for acute care DO patients [6,7].

Although patients with DO are frequently admitted to a hospital, often no specific treatment is provided except for close observation of their vital signs and level of

*Address correspondence to this author at the Department of Emergency and Critical Care Medicine, St. Luke's International Hospital, 9-1 Akashicho, Chuo-city, Tokyo, 104-8560, Japan; Tel: +81 (03) 3541-5151; Fax: +81 (03) 5550-2051; E-mail: mican-philia@sunny.ocn.ne.jp consciousness [8]. The drugs such as benzodiazepines do not usually damage vital organs of patients, although they lower a level of their consciousness by suppressing central nervous systems [5]. Recent evidence-based recommendations for treatment of DO patients suggested respiratory support and tracheal intubation with mechanical ventilation for patients with respiratory suppression and no gastric lavage or ipecac emetics for most patients $[9,10]$. Indeed, if admitted without complications, many DO patients will be discharged in a few days. However, the individual variations for durations of this medical observation are large [11]. Large costs and medical resources are needed for caring these patients with longer hospital stay [12-14].

Previous studies have developed the models to predict whether those patients would need inpatient admission or would need specific treatment $[15,16]$. However, there have been few studies to investigate risk factors associated with prolonged length of hospital stay (LOS) in patients who admitted to hospitals from ED. Thus, our aim in this study was to identify significant risk factors influencing a length of hospital stay in DO patients based on initial clinical data including demographics, vital and physical signs, and simple laboratory data. To our knowledge, this may be the first study to determine significant risk factors for prolonged LOS for DO patients. 


\section{MATERIAL AND METHODOLOGY}

\section{Study Subjects}

We reviewed the medical records of 140 consecutive DO patients who were admitted to St. Luke's International Hospital in Tokyo, Japan, from July 1, 2005 to July 31, 2006. This institution is an urban community teaching hospital which provides primary to tertiary care to a population of about 97 thousands. The inclusion criteria for study subjects were: 1) DO of a psychotropic drug as defined as any drug capable of affecting the mind, emotions, and behavior, 2) inpatient admission through the ED. The exclusion criteria were: 1) poisoning from pesticide, insecticide, toxic gas, carbon monoxide, fumes, hydrocarbons, and other chemical substances, 2) multiple trauma, 3) cardiopulmonary arrest on arrival to the ED, and 3) iatrogenic drug overdose. Prior approval from the Institutional Review Board of St. Luke's International Hospital was obtained.

\section{Data Collection}

We collected data from the emergency department data registry and in-patients' medical record with discharge diagnosis of DO. We identified the ingested psychotropic drugs also from the medical charts. The clinical staff in this hospital collected data for the drug ingredients by analyzing and counting the number of empty PTP (Press through Package) sheets of the drugs. We collected data for demographics, initial vital signs (blood pressure [BP], heart rate [HR], respiratory rate, and body temperature) and Glasgow Coma Scale (GCS). Initial laboratory data (standard protocol for altered mental status) included complete blood count (white blood count [WBC], hemoglobin, and hematocrit), blood chemistry (sodium, potassium, and creatinine), arterial blood gas $\left(\mathrm{pH}, \mathrm{PaCO}_{2}\right.$, and $\left.\mathrm{PaO}_{2}\right)$. The main outcome measure was inpatient LOS in this study and was calculated by subtracting day of admission from day of discharge.

\section{Statistical Analysis}

We calculated descriptive statistics for demographics, vital signs and physical findings and laboratory data. We constructed a stepwise multivariable adjusted linear regression model to determine significant factors for a prolonged LOS. A stepwise procedure was involved with likelihood ratio test methods with a variable entry at $p<0.05$ and a variable exclusion at $p>0.10$. Beta coefficients of each covariate were determined with $95 \%$ confidence intervals $(\mathrm{CI})$ for each unit change of continuous variables. For blood leukocyte, we divided the count per $\mathrm{mm}^{3}$ by 100 to obtain comparative values for a beta coefficient.

Next, we performed a recursive partitioning analysis to determine the most important variable among the significant factors for a prolonged hospital stay of 3 days or greater. Ten-fold cross validation method was used for internal validation of the classification tree. All $\mathrm{p}$ values were two sided and $\mathrm{p}<0.05$ was considered statistically significant. All analyses were performed using SPSS version 15.0J (SPSSJapan, Tokyo, Japan).

\section{RESULTS}

We identified a total of 140 patients with intentional DO who were admitted to the hospital. The mean age was 35.3 with a standard deviation of 14.8. Seventy-eight percent were women. Ninety-seven percent of these patients were transported to the ED by ambulance. Table 1 shows the frequently ingested psychotropic drugs including: benzodiazepines $(n=104)$; phenothiazines $(n=34)$; selective serotonin reuptake inhibitors $(\mathrm{n}=23)$; non-benzodiazepine sedatives $(\mathrm{n}=20)$; tricyclic antidepressants $(\mathrm{n}=18)$; barbiturates $(\mathrm{n}=16)$; anticholinergics $(\mathrm{n}=16)$; benzamides $(\mathrm{n}=14)$; antihistamines $(\mathrm{n}=14)$; serotonin dopamine antagonists $(\mathrm{n}=$ 11); serotonin-noradrenaline reuptake inhibitor $(\mathrm{n}=10)$; and antipsychotics $(\mathrm{n}=10)$. Some patients had ingested more than one type of these medications. A few patients had imbibed alcoholic beverages along with these medications.

Table 1. Psychotropic Drugs Overdosed by Admitted Patients

\begin{tabular}{|l|c|}
\hline \multicolumn{1}{|c|}{ Drug Classification } & n \\
\hline \hline Benzodiazepines & 104 \\
\hline Phenothiazines & 34 \\
\hline Selective serotonin reuptake inhibitors & 23 \\
\hline Non-benzodiazepine sedatives & 20 \\
\hline Tricycle & 18 \\
\hline Barbiturates & 16 \\
\hline Anticholinergic drugs & 16 \\
\hline Bezamides & 14 \\
\hline Antihistamines & 14 \\
\hline Serotonin dopamine antagonists & 11 \\
\hline Serotonin-noradrenaline reuptake inhibitors & 10 \\
\hline Antipsychotics & 10 \\
\hline Sodium valproate & 9 \\
\hline Antidepressants & 7 \\
\hline Butyrophenones & 2 \\
\hline Carbamazepine & 2 \\
\hline Tetracyclic & 2 \\
\hline Bromoisovalerylureas & 2 \\
\hline Methylphenidates & 2 \\
\hline Amphetamines & 2 \\
\hline Opioids & 2 \\
\hline Some patients ingested more than one type of the drug & 2 \\
\hline \multicolumn{1}{|c|}{} & 2 \\
\hline
\end{tabular}

* Some patients ingested more than one type of the drug.

Table 2 presents clinical characteristics of patients with DO. The mean Glasgow Coma Scale was 10 with a standard deviation of 4.4. The mean length of hospital stay was 2.87 days with a range of 1 to 17 . Seventy-nine percent (110/140) of patients were discharged from the hospital within 3 days, while $6 \%(8 / 140)$ of patients were hospitalized for 7 days or more. Most patients were discharged from the hospital soon after restoration of their usual consciousness levels (Glasgow Coma Scale $=15$ ) along with the follow-up psychiatric outpatient appointments.

Systemic complications occurred in 12 of 140 patients (8.6\%). They included: aspiration pneumonia in 6 patients; airway compromise or respiratory suppression in 4; acute 
renal failure in 2; and, severe rhabdomyolysis in 1. All patients discharged within 3 days after the admission remained asymptomatic for the following month.

Table 2. Clinical Charactaristics of Patients with Psychotropic Drug Overdose

\begin{tabular}{|c|c|c|c|}
\hline Characteristic & \multicolumn{3}{|c|}{$N=140$} \\
\hline \multicolumn{4}{|l|}{ Demographics } \\
\hline Age, mean \pm SD & 35.3 & \pm & 14.8 \\
\hline Male gender, $\%$ & 22.1 & & \\
\hline \multicolumn{4}{|l|}{ Vital signs, mean $\pm \mathrm{SD}$} \\
\hline Systolic blood pressure, $\mathrm{mmHg}$ & 112.6 & \pm & 20.7 \\
\hline Diastolic blood pressure, $\mathrm{mmHg}$ & 68.2 & \pm & 13.8 \\
\hline Mean blood pressure, $\mathrm{mmHg}$ & 83 & \pm & 15.1 \\
\hline Heart rate, $/ \mathrm{min}$ & 83 & \pm & 21 \\
\hline Respiratory rate, /min & 18.9 & \pm & 4.9 \\
\hline Temperature, centigrade & 36 & \pm & 1 \\
\hline Glasgow coma scale, mean $\pm \mathrm{SD}$ & 10 & \pm & 4.4 \\
\hline \multicolumn{4}{|l|}{ Complete blood count, mean \pm SD } \\
\hline Leukocyte count, $/ \mathrm{mm}^{3}$ & 8213.6 & \pm & 3913.2 \\
\hline Hemoglobin, $\mathrm{g} / \mathrm{dl}$ & 13.1 & \pm & 1.7 \\
\hline Hematocrit, \% & 38.5 & \pm & 5.6 \\
\hline \multicolumn{4}{|l|}{ Blood chemistry, mean \pm SD } \\
\hline Sodium, mEq/L & 140.6 & \pm & 2.8 \\
\hline Potassium, mEq/L & 3.8 & \pm & 0.4 \\
\hline Creatinine, $\mathrm{mEq} / \mathrm{L}$ & 0.87 & \pm & 1.27 \\
\hline \multicolumn{4}{|l|}{ Arterial Blood gas, mean \pm SD } \\
\hline $\mathrm{pH}$ & 7.378 & \pm & 0.0648 \\
\hline $\mathrm{PaCO}_{2}, \mathrm{mmHg}$ & 42.9 & \pm & 8.7 \\
\hline $\mathrm{PaO}_{2}, \mathrm{mmHg}$ & 157.4 & \pm & 116.1 \\
\hline Lengths of Hospital Stay, days, mean \pm SD & 2.87 & \pm & 2.41 \\
\hline
\end{tabular}

Using a stepwise linear regression model, significant risk factors of prolonged LOS were the following (Table 3): 1) older age (beta coefficient $0.049 ; 95 \% \mathrm{CI}, 0.026,0.071$ ), 2) increased heart rate (beta $0.020 ; 95 \% \mathrm{CI}, 0.002,0.038$ ), 3) lower diastolic blood pressure (beta $-0.062 ; 95 \% \mathrm{CI},-0.087$, $0.038), 4$ ) lower Glasgow Coma Scale (beta $-0.094 ; 95 \% \mathrm{CI}$, $-0.170,-0.019$ ), and higher leukocyte count $/ 100$ (beta 0.014 ; $95 \% \mathrm{CI}, 0.004,0.023)$. We constructed a prediction model including the above parameters along with an intercept of 3.517 in a multiple linear regression. The final model was as follows:

Predicted length of hospital stay (days) $=$

$3.517+($ age $\times 0.049)+(\mathrm{HR} \times 0.020)-(\mathrm{DBP} \times 0.062)-(\mathrm{GCS} \times$ $0.094)$

$+[(\mathrm{WBC} / 100) \times 0.014]$

$\mathrm{DBP}=$ diastolic blood pressure

The adjusted $\mathrm{R}$ square of this model was $0.391 \pm 1.839$ (standard deviation). The mean WBC in patients with aspiration pneumonia was 14,667 , which was higher than that of patients without aspiration pneumonia.

Table 4 presents distributions of the significant risk factors influencing LOS in DO patients based on the mean number of LOS (3 days). Based on a recursive partitioning analysis with internal cross-validation, $\mathrm{WBC} \geq 12,900$ was identified as a cutoff level at risk for prolonged LOS of $\geq 3$ days. Among the high risk group with WBC $\geq 12,900$ ( $\mathrm{n}=$ $13 ; 9.4 \%$ of all patients), $69.2 \%$ of them had stayed for more than 3 days in the hospital. Among the low risk group with WBC of $\leq 12,900$ ( $n=125 ; 90.6 \%$ of all patients), only $16.8 \%$ of had stayed for more than 3 days.

\section{DISCUSSION}

The results of our study indicated that there was an increased risk for a prolonged hospital admission in patients with DO who present with the following clinical findings: older age, increased heart rate, lower blood pressure, altered mental status and elevated WBC. Among these clinical variables, increased WBC may be used as the most important predictor for a prolonged LOS. Since the cause of a longer hospitalization in these patients is usually related to various complications, these risk factors may be considered as factors associated with the increased complication rate in patients with psychotropic DO.

Although DO is commonly recognized among the young people in Japan as well as in other countries [17], we found that older age was a risk factor for a prolonged LOS in the

Table 3. Multiple Linear Regression for the Length of Hospital Stay ( $=140)$

\begin{tabular}{|c|c|c|c|c|}
\hline & \multirow{2}{*}{$\frac{\text { Beta }}{\text { Coefficient }}$} & \multicolumn{2}{|c|}{$95 \%$ CI } & \multirow{2}{*}{ p-Value } \\
\hline & & Lower & Upper & \\
\hline Intercept & 3.517 & 1.143 & 5.892 & 0.004 \\
\hline Age & 0.049 & 0.026 & 0.071 & $<0.0001$ \\
\hline Heart rate & 0.020 & 0.002 & 0.038 & 0.029 \\
\hline Diastolic blood pressure & -0.062 & -0.087 & -0.038 & $<0.0001$ \\
\hline Glasgow coma scale & -0.094 & -0.170 & -0.019 & 0.015 \\
\hline Blood leukocyte count / 100 & 0.014 & 0.004 & 0.023 & 0.005 \\
\hline
\end{tabular}


geriatric population, likely due to increased concentration of the drug from a delayed clearance and its longer half-life from age-related renal hypo-function and from increased percentage of body adipose tissue. Moreover, central nervous system capacity to regain a recovery of the consciousness in older persons may be lower than that of the young. One report in Japan also found that patients with DO over 45 years old may be at risk for a completed suicide than the younger [13].

Table 4. Distributions of Risk Factors for the Length of Hospital Stay $(\mathrm{N}=140)$

\begin{tabular}{|l|c|c|c|c|}
\hline \multirow{2}{*}{} & \multicolumn{2}{|c|}{$\begin{array}{c}\text { LOS }=<3 \\
\text { Days }(\mathbf{n}=\mathbf{1 1 0})\end{array}$} & \multicolumn{2}{c|}{$\begin{array}{c}\text { OOS }>3 \text { Days } \\
(\mathbf{n}=\mathbf{3 0})\end{array}$} \\
\cline { 2 - 5 } & Mean & SD & Mean & SD \\
\hline \hline Age, yr & 34.5 & 13.5 & 38.1 & 18.7 \\
\hline Heart rate, /min & 79.8 & 17.7 & 94.9 & 27.6 \\
\hline Diastolic blood pressure, $\mathrm{mmHg}$ & 69.4 & 12.5 & 63.7 & 17.4 \\
\hline Glasgow coma scale & 10.5 & 4.2 & 8.2 & 4.7 \\
\hline Blood leukocyte count $/ \mathrm{mm}^{3} / 100$ & 75.5 & 30.7 & 106.5 & 54.9 \\
\hline
\end{tabular}

The results of this study also indicated that increased HR at admission was a risk factor for a prolonged LOS. The increased HR may reflect the presence of systemic complications such as dehydration or concomitant infection. In addition, this may be an indicator for the cardiotoxic effects from the ingested drug itself. Both the systemic complications and the cardiotoxic states may explain the delayed recovery in these patients. The prediction rules developed in previous studies for patients with opioid DO also identified abnormal HR including both tachycardia and bradycardia as an increased risk for admission [18].

Lower BP at admission was a risk factor for a prolonged LOS in this study. It may reflect the presence of systemic complications such as hypovolemia with concomitant systemic inflammatory response syndrome or sepsis. In addition, lower BP may be an indicator of the possible cardiovascular depressive effects from the ingested drugs [15].

Low conscious level at admission was a risk factor for a prolonged LOS in this study. Patients with an impaired level of consciousness tended to have the more severe central nervous system dysfunction [19]. Comatose or semicomatose states may increase the risk for developing systemic complications including aspiration pneumonia. A previous study in Canada also identified an altered consciousness level as one of the predictors for admission to hospitals in patients with opioid overdose [18].

Elevated $\mathrm{WBC}$ at admission was a risk factor for a prolonged LOS in this study. Higher WBC may indicate the presence of systemic inflammation including aspiration pneumonia or other infectious complications. The admission WBC count may be a surrogate indicator for the presence of infectious complications which may warrant further workup for unsuspected infections. Alternatively, higher WBC may be caused by the more severe degree of stress from DO.
Although DO with psychotropics having cardiotoxic effects are among the most common causes of death [20,21], the majority of deaths occur out of hospital [22]; consequently, in-hospital mortality rate is generally low, less than $2 \%$ [23-25]. Indeed, of 140 hospitalized patients with DO in this study, none died.

Prediction for LOS in patients with psychotropic DO is not simple. The hospitalization period in DO patients may be determined not only by central nervous system suppression effects from the DO itself, but also by the accompanied systemic complications. For individual complications in this study, aspiration pneumonia was the most common. Higher WBC can be a surrogate indicator for the presence of aspiration pneumonia at the time of admission.

The prevalence of DO creates significant demands on a healthcare system. In 2005 the Tokyo Fire Department reported 7152 patients with psychotropic DO over the metropolitan Tokyo area. Many of them were transported and admitted to acute care hospitals. During the same year, 171 drug overdose patients came to our facility by ambulance and private conveyance and 140 patients were admitted. During the same period the occupancy rate for the hospital approached $100 \%$. Thus, despite the relatively short LOS for DO patients, a significant burden is placed on healthcare resources. The prediction rule developed in this study may be utilized for effective allocation of available inpatient beds. Low risk patients can safely be admitted to observation units of an emergency department for short-term observation.

There are a few limitations in this study. First, information on ingested drugs may not be inaccurate in terms of the ingredients and the quantity of the drugs. Accuracy can be improved by using urine toxicology screening. Second, we did not analyze the baseline mental state as a potential risk factor for prolonged LOS in this study. Psychotic patients may be at a higher risk for prolonged LOS than nonpsychotic patients.

\section{CONCLUSION}

Hospitalized patients with DO in a community teaching hospital in Japan have a fair discharge survival. Risk factors for a prolonged LOS in these patients included older age, increased HR, lower BP, altered mental status and elevated WBC. LOS may be predicted using a model developed from these simple clinical data. Alternatively, WBC could be used as a simple and quick tool. Further studies are needed to confirm the results of this study by external validation.

\section{ACKNOWLEDGMENTS}

We thank all attending physicians and residents caring patients with drug overdose in our hospital.

\section{REFERENCES}

[1] Blanc PD, Jones MR, Olson KR. Surveillance of poisoning and drug overdose through hospital discharge coding, poison control center reporting, and the Drug Abuse Warning Network. Am J Emerg Med 1993; 11(1): 14-9.

[2] Hawton K, Fagg J. Trends in deliberate self poisoning and self injury in Oxford, 1976-90. BMJ 1992; 304(6839): 1409-11.

[3] Wazaify M, Kennedy S, Hughes CM, McElnay JC. Prevalence of over-the-counter drug-related overdoses at Accident and Emergency departments in Northern Ireland--a retrospective evaluation. J Clin Pharm Ther 2005; 30(1): 39-44. 
[4] Epstein FB. Fatal benzodiazepine toxicity? Am J Emerg Med 1987; 5(5): 472-3.

[5] Greene SL, Dargan PI, Jones AL. Acute poisoning: understanding 90\% of cases in a nutshell. Postgrad Med J 2005; 81(954): 204-16.

[6] Greaves I, Goodacre S, Grout P. Management of drug overdoses in accident and emergency departments in the United Kingdom. J Accid Emerg Med 1996; 13(1): 46-8.

[7] Gunnell D, Bennewith O, Peters TJ, House A, Hawton K. The epidemiology and management of self-harm amongst adults in England. J Public Health (Oxf) 2005; 27(1): 67-73.

[8] Stein MD, Bonanno J, O'Sullivan PS, Wachtel TJ. Changes in the pattern of drug overdoses. J Gen Intern Med 1993; 8(4): 179-84.

[9] Krenzelok EP, McGuigan M, Lheur P. Position statement: ipecac syrup. American Academy of Clinical Toxicology; European Association of Poisons Centres and Clinical Toxicologists. J Toxicol Clin Toxicol 1997; 35(7): 699-709.

[10] Vale JA. Position statement: gastric lavage. American Academy of Clinical Toxicology; European Association of Poisons Centres and Clinical Toxicologists. J Toxicol Clin Toxicol 1997; 35(7): 711-9.

[11] Chan TY, Critchley JA. Hospital admissions due to acute poisoning in the New Territories, Hong Kong. Southeast Asian J Trop Med Public Health 1994; 25(3): 579-82.

[12] Hatta K. [Triage guideline for suicidal patients with acute poisoning and drug overdoses]. Chudoku Kenkyu 2005; 18(2): 137-40.

[13] Hamanaka S. [Lethal suicide attempts and parasuicide in drug overdose patients]. Chudoku Kenkyu 2005; 18(2): 123-6.

[14] Nakamura M, Tanmachi K, Okumura M, Takesawa K, Ichinose K. [Self-poisoning repeater--psychiatric considerations]. Chudoku Kenkyu 2005; 18(2): 127-36.

[15] Meulendijks CF, van den Berg EJ, Fortuyn HD, Verkes RJ, van der Wilt GJ, Kramers C. Predicting the need for hospital admission in patients with intentional drug overdose. Neth J Med 2003; 61(5): 164-7.

[16] Hollander JE, McCracken G, Johnson S, Valentine SM, Shih RD. Emergency department observation of poisoned patients: how long is necessary? Acad Emerg Med 1999; 6(9): 887-94.

[17] Olfson M, Gameroff MJ, Marcus SC, Greenberg T, Shaffer D. Emergency treatment of young people following deliberate selfharm. Arch Gen Psychiatry 2005; 62(10): 1122-8.

[18] Christenson J, Etherington J, Grafstein E, et al. Early discharge of patients with presumed opioid overdose: development of a clinical prediction rule. Acad Emerg Med 2000; 7(10): 1110-8.

[19] Bouknight RR, Alguire PC, Lofgren RP, Hoppe RB. Selfpoisoning: outcome and complications in the community hospital. J Fam Pract 1986; 23(3): 223-5.

[20] Crome P. The toxicity of drugs used for suicide. Acta Psychiatr Scand Suppl 1993; 371: 33-7.

[21] Buckley NA, Whyte IM, Dawson AH, McManus PR, Ferguson NW. Correlations between prescriptions and drugs taken in selfpoisoning. Implications for prescribers and drug regulation. Med $\mathrm{J}$ Aust 1995; 162(4): 194-7.

[22] Kapur N, Turnbull P, Hawton K, et al. Self-poisoning suicides in England: a multicentre study. QJM 2005; 98(8): 589-97.

[23] Callaham M, Kassel D. Epidemiology of fatal tricyclic antidepressant ingestion: implications for management. Ann Emerg Med 1985; 14(1): 1-9.

[24] Buckley NA, Dawson AH, Whyte IM, Henry DA. Greater toxicity in overdose of dothiepin than of other tricyclic antidepressants. Lancet 1994; 343(8890): 159-62.

[25] Strom J, Sloth Madsen P, Nygaard Nielsen N, Bredgaard Sorensen M. Acute self-poisoning with tricyclic antidepressants in 295 consecutive patients treated in an ICU. Acta Anaesthesiol Scand 1984; 28(6): 666-70

(C) Abe et al.; Licensee Bentham Open.

This is an open access article distributed under the terms of the Creative Commons Attribution License (http://creativecommons.org/license/by/2.5/), which permits unrestrictive use, distribution, and reproduction in any medium, provided the original work is properly cited. 